\title{
Nodal Quasiparticle Lifetimes in Cuprate Superconductors
}

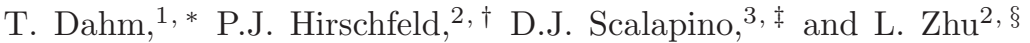 \\ ${ }^{1}$ Institut für Theoretische Physik, Universität Tübingen, Tübingen, Germany \\ ${ }^{2}$ Physics Department, University of Florida, Gainesville, FL 32611 USA \\ ${ }^{3}$ Department of Physics, University of California, Santa Barbara, CA 93106-9530 USA
}

(Dated: November 5, 2018)

\begin{abstract}
A new generation of angular-resolved photoemission spectroscopy (ARPES) measurements on the cuprate superconductors offer the promise of enhanced momentum and energy resolution. In particular, the energy and temperature dependence of the on-shell nodal $\left(k_{x}=k_{y}\right)$ quasiparticle scattering rate can be studied. In the superconducting state, low temperature transport measurements suggest that one can describe nodal quasiparticles within the framework of a BCS $d$-wave model by including forward elastic scattering and spin-fluctuation inelastic scattering. Here, using this model, we calculate the temperature and frequency dependence of the on-shell nodal quasiparticle scattering rate in the superconducting state which determines the momentum width of the ARPES momentum distribution curves. For a zero-energy quasiparticle at the nodal momentum $k_{N}$, both the elastic and inelastic scattering rate show a sudden decrease as the temperature drops below $T_{c}$, reflecting the onset of the gap amplitude. At low temperatures the scattering rate decreases as $T^{3}$ and approaches a zero temperature value determined by the elastic impurity scattering. For $T>T_{c}$, we find a quasilinear dependence on $T$. At low reduced temperatures, the elastic scattering rate for the nodal quasiparticles exhibits a quasilinear increase at low energy $\omega$ which arises from elastic scattering processes. The inelastic spin-fluctuation scattering leads to a low energy $\omega^{3}$ dependence which, for $\omega \gtrsim 3 \Delta_{0}$, crosses over to a quasilinear behavior.
\end{abstract}

PACS numbers: 74.25.Jb, 74.20.Fg, 74.72.-h

\section{INTRODUCTION}

A $d$-wave BCS framework has proved useful in describing the thermal 1, 2] and microwave 3] conductivities of the superconducting cuprates [4, 5, 6]. This suggests that a quasiparticle description of the nodal excitations is adequate, at least at low energies. Here, the frequencies are small compared with the gap magnitude and the temperature dependence of the nodal quasiparticle lifetime plays the dominant role. Recent angle-resolved photoemission spectroscopy (ARPES) experiments on $\mathrm{Bi}_{2} \mathrm{Sr}_{2} \mathrm{Ca}_{1} \mathrm{Cu}_{2} \mathrm{O}_{8}$ (BSCCO) are providing high resolution data on the momentum and energy dependence of the nodal quasiparticle lifetimes as well as their temperature dependence in this low energy range $7,[8,9,10]$. Thus, the question arises whether transport and ARPES data can be understood within a single framework. Establishing this agreement is important to confirming the nature of the superconducting phase as a BCS-like $d$-wave state with nodal quasiparticle excitations.

The width $\Delta k(\omega, T)$ of the momentum distribution curve (MDC) measured by ARPES experiments is proportional to the inverse of the on-shell quasiparticle lifetime. Along a nodal cut in momentum, with $k_{x}=k_{y}$, the magnitude $k=\sqrt{k_{x}^{2}+k_{y}^{2}}$ is set by $\omega=\varepsilon_{k}$, i.e. $k$

\footnotetext{
*Electronic address: thomas.dahm@uni-tuebingen.de

${ }^{\dagger}$ Electronic address: pjh@phys.ufl.edu

${ }^{\ddagger}$ Electronic address: djs@vulcan2.physics.ucsb.edu

$\S$ Electronic address: zly@phys.ufl.edu
}

becomes effectively a function of $\omega$; we refer to this as "on-shell". Here, $\varepsilon_{k}$ is the quasiparticle energy. Thus, the lifetime of such a nodal quasiparticle depends upon $\omega$ and the temperature $T$. As $k$ decreases from the nodal Fermi momentum $k_{N}, \omega$ can be swept over an energy range which is significantly larger than $\Delta_{0}$. Therefore, there is a need to extend the inelastic scattering lifetime calculations to cover a wider range of energies. In addition, impurity scattering, particularly due to out-of-plane forward scattering, is believed to be important so that it is also of interest to determine its temperature and energy dependence 11, 12].

In the following, we will find it convenient to discuss the elastic and inelastic quasiparticle scattering in terms of a scattering rate

$$
\Gamma(\omega, \mathbf{k}, T)=-\Sigma^{\prime \prime}(\omega, \mathbf{k}, T),
$$

where $\Sigma^{\prime \prime}$ is the imaginary part of the quasiparticle self-energy. The inverse of the quasiparticle lifetime $1 / \tau(\omega, \mathbf{k}, T)=2 \Gamma(\omega, \mathbf{k}, T)$ and the width of the MDC about a given $\mathbf{k}$ determined from $\varepsilon_{k}=\omega$ is

$$
\Delta k(\omega, T)=\frac{2 \Gamma\left(\omega,\left.\mathbf{k}\right|_{\omega=\varepsilon_{\mathbf{k}}}, T\right)}{v(\mathbf{k})},
$$

with $v(\mathbf{k})=\frac{\partial \varepsilon_{\mathbf{k}}}{\partial \mathbf{k}}$ the bare band velocity. Here, we report on results obtained within a $d$-wave BCS framework for the energy and temperature dependence of the nodal quasiparticle elastic and inelastic scattering rates and discuss the $\omega$ and $T$ dependence of $\Gamma\left(\omega,\left.\mathbf{k}\right|_{\omega=\varepsilon_{k}}, T\right)$.

We begin in Section II with a discussion of the temperature and energy dependence of the elastic impurity scattering contribution. In BSCCO, there are both in-plane 
and out-of-plane scattering centers [13]. The in-plane impurities are believed to give rise to strong scattering and are often treated in the unitary limit 14]. On the other hand, the out-of-plane impurity scattering is weak and tends to be forward. It will be treated within a selfconsistent Born approximation. We will see that the temperature dependence of the elastic scattering rate arises from the opening of the gap as $T$ decreases below $T_{c}$, while its energy dependence reflects the decreased phase space as $\omega$ becomes smaller than the gap magnitude. The low energy inelastic scattering discussed in Section III is dominated by short range Coulomb scattering. At low energies, the linear $\omega$ dependence of the density of states gives rise to an inelastic scattering rate which varies as $T^{3}$ for $T$ larger than $\omega$ or $\omega^{3}$ for $\omega$ larger than $T$. At higher energies, one can probe the $\omega$-dependence of the effective interaction. Here, we confine ourselves to the Hubbard model and explore the spin-fluctuation contributions to the higher energy behavior of the inelastic scattering. In Section IV, we discuss the combined effects of the elastic and inelastic scattering processes, and Section V contains our conclusions.

\section{ELASTIC SCATTERING}

Elastic scattering in BSCCO can arise from both impurities and disorder in the $\mathrm{CuO}$ planes as well as from regions outside these planes. The scattering rate due to unitary scatterers (possibly $\mathrm{Cu}$ vacancies) with concentration roughly $n_{u} \sim 0.2 \%$ observed as zero-bias resonances in scanning tunneling microscopy (STM) experiments 14 is well understood theoretically. It will be treated as usual in the self-consistent $T$-matrix approximation,

$$
\underline{\Sigma}_{e l, u}=-\frac{n_{u}}{\sum_{\mathbf{k}} G(\mathbf{k}, \omega)} \tau_{0},
$$

where $\tau_{i}$ are the $2 \times 2$ Pauli matrices in Nambu notation. At low frequencies and temperatures, the unitary scatterers give rise to an impurity resonance of height $\Sigma_{e l, u}^{\prime \prime}(\omega=0) \equiv \gamma_{u} \simeq \sqrt{\Gamma_{u} \Delta_{0}} \sim 10^{-2} t$, and roughly the same width, with $t$ the nearest-neighbor hopping matrix element. In the normal state we find a scattering rate of $\Gamma_{u} \sim n_{u} E_{F} \simeq 10^{-3} t$ due to these defects, leading to $\gamma_{u} \simeq 1$ to $2 \mathrm{meV}$. If, as we believe, the normal state elastic scattering rate at the node is a significant fraction of the gap, the contribution of the unitary scatterers will be difficult to resolve in ARPES experiments on BSCCO.

The elastic scattering from out-of-plane impurities, such as interstitial $\mathrm{O}$ ions, can be modeled by a momentum-dependent potential,

$$
V\left(\mathbf{k}, \mathbf{k}^{\prime}\right)=V_{0} f\left(\mathbf{k}, \mathbf{k}^{\prime}\right)
$$

which has a forward scattering form factor $f\left(\mathbf{k}, \mathbf{k}^{\prime}\right)$ which cuts off the scattering when $\left|\mathbf{k}-\mathbf{k}^{\prime}\right|$ exceeds a momentum $\kappa$, as has been discussed in Ref. 12. We will measure $\kappa$ in units of $k_{F}$ so that $\kappa^{-1}$ characterizes the range of the impurity potential in units proportional to the $\mathrm{Cu}-\mathrm{Cu}$ spacing $a$. Since the scattering from an out-of-plane impurity is relatively weak, it can be treated within the selfconsistent Born approximation [15]. For a nodal quasiparticle with $k_{x}=k_{y}$, the gap vanishes and the scattering rate due to elastic scattering is determined from the imaginary part of the $\tau_{0}$ Nambu self-energy in the self-consistent Born approximation,

$$
\Sigma_{e l, 0}(\mathbf{k}, \omega)=n_{i} \sum_{k^{\prime}}\left|V\left(\mathbf{k}, \mathbf{k}^{\prime}\right)\right|^{2} \frac{\tilde{\omega}}{\tilde{\omega}^{2}-\varepsilon_{k^{\prime}}^{2}-\tilde{\Delta}_{k^{\prime}}^{2}},
$$

where $n_{i}$ is the planar density of the out-of-plane impurities. In addition, because the potential $V\left(\mathbf{k}, \mathbf{k}^{\prime}\right)$ is anisotropic, it is necessary to renormalize the order parameter as well:

$$
\Sigma_{e l, 1}(\mathbf{k}, \omega)=n_{i} \sum_{k^{\prime}}\left|V\left(\mathbf{k}, \mathbf{k}^{\prime}\right)\right|^{2} \frac{\tilde{\Delta}_{\mathbf{k}^{\prime}}}{\tilde{\omega}^{2}-\varepsilon_{k^{\prime}}^{2}-\tilde{\Delta}_{k^{\prime}}^{2}},
$$

where $\tilde{\omega}=\omega-\Sigma_{e l, 0}$ and $\tilde{\Delta}_{\mathbf{k}}=\Delta_{\mathbf{k}}+\Sigma_{e l, 1}$. The renormalization of $\varepsilon_{\mathbf{k}}$ by disorder is also nonzero in general but was shown to be negligible in Ref. 12, and will therefore be neglected here. For our numerical calculations we will use a simple parameterization of the band structure with

$$
\varepsilon_{k}=-2 t\left(\cos k_{x}+\cos k_{y}\right)-4 t^{\prime} \cos k_{x} \cos k_{y}-\mu
$$

and

$$
\Delta_{k}=\frac{\Delta_{0}}{2}\left(\cos k_{x}-\cos k_{y}\right) .
$$

Here, $t^{\prime}=-0.35$ and $\mu=-1.1$, with energy measured in units of nearest neighbor hopping $t$. For the temperature dependence of the gap we use a common interpolation form

$$
\Delta_{0}(T)=\Delta_{0} \tanh \left(\alpha \sqrt{\frac{T_{c}}{T}-1}\right)
$$

with $\alpha=3,2 \Delta_{0} / k T_{c}=6$, and $\Delta_{0}=0.2$. These parameters are chosen to give the observed magnitude of the $T=0$ gap and a somewhat more rapid rise at $T_{c}$, consistent with experiment.

The scattering rate $\Gamma_{e l}\left(\mathbf{k}_{N}, \omega\right)$ is now determined by the imaginary part of the solution of Eq. (5) at the nodal wavevector $\mathbf{k}_{N}$. Before turning to a numerical evaluation of Eq. (5)-(6), we consider a simple analytic approximation similar in spirit to that discussed in [17] in the context of $T_{c}$ suppression by disorder [18]. Measuring the momentum transfer from $\mathbf{k}_{N}$ along the $k_{\perp}$ and $k_{\|}$coordinates shown in Fig. प the imaginary part of Eq. (5) can be written as

$\Gamma_{e l}=-n_{i}\left|V_{0}\right|^{2} \operatorname{Im} \int \frac{d k_{\|}^{\prime} d \mathbf{k}_{\perp}^{\prime}}{(2 \pi)^{2}}\left|f\left(\mathbf{k}, \mathbf{k}^{\prime}\right)\right|^{2} \frac{\tilde{\omega}}{\tilde{\omega}^{2}-v_{1}^{2} k_{\perp}^{\prime 2}-\tilde{v}_{2}^{2} k_{\|}^{\prime 2}}$ 


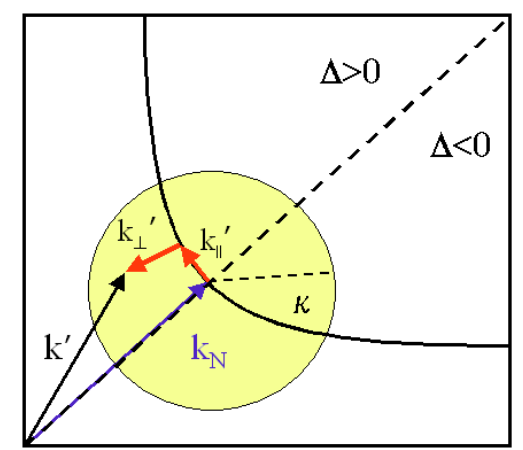

FIG. 1: (Color online) The Fermi surface corresponding to the band $\varepsilon_{k}$ given by Eq. (7) with $t^{\prime} / t=-0.3$ and $\mu=-1.1$. The difference of the scattered wave vector $\mathbf{k}^{\prime}$ from the nodal wave vector $\mathbf{k}_{N}$ is shown as its parallel displacement along the Fermi surface $\mathbf{k}_{\|}$and the perpendicular displacement $\mathbf{k}_{\perp}$.

Here, $v_{1}=v_{F}$ at the nodal point and $\tilde{v}_{2}$ is the renormalized gap velocity. We have assumed that the $\kappa$ cut-off prevents scattering to other nodes.

Since $v_{1} \gg v_{2}$, the $\kappa$ cut-off primarily affects the $k_{\|}$ integral and one can integrate freely over the important range $k_{\perp}$ giving

$$
\Gamma_{e l}=\frac{1}{2} n_{i} N_{0}\left|V_{0}\right|^{2} \operatorname{Re} \int_{-\kappa}^{\kappa} d k_{\|}^{\prime} \frac{\tilde{\omega}}{\sqrt{\tilde{\omega}^{2}-\tilde{v}_{2}^{2} k_{\|}^{\prime 2}}}
$$

with $N_{0}=\left(2 \pi v_{F}\right)^{-1}$ the band density of states in units in which $k_{F}=1$. Setting $\tilde{\omega}=\omega+i \Gamma_{e l}$, i.e. neglecting the real part of the self-energy, this becomes

$$
\Gamma_{e l}=\frac{\Gamma_{0}}{\tilde{v}_{2} \kappa} \operatorname{Re}\left[\left(\omega+i \Gamma_{e l}\right) \sin ^{-1}\left(\frac{\tilde{v}_{2} \kappa}{\omega+i \Gamma_{e l}}\right)\right],
$$

where $\Gamma_{0}=n_{i} N_{0}\left|V_{0}\right|^{2} \kappa$ is the normal state elastic scattering rate and $\tilde{v}_{2} \kappa$ is the maximum renormalized order parameter probed by the scattering. From this result we recover immediately the $\omega \rightarrow 0, k_{\|} \rightarrow 0$ limit that $\Gamma_{e l} \rightarrow \Gamma_{0}$ from Ref. 12 .

The gap velocity at the node in the pure system, $v_{2} \simeq$ $2 \Delta_{0} / k_{N}$, is significantly renormalized in the presence of disorder to $\tilde{v}_{2}$. From Eq. (6) we find, for $k_{\|} \ll \kappa$,

$$
\left(\tilde{v}_{2}-v_{2}\right) k_{\|}=\frac{\Gamma_{0}}{2 \kappa} \operatorname{Im} \int_{-\kappa}^{\kappa} d k_{\|}^{\prime} \frac{\tilde{v}_{2}\left(k_{\|}^{\prime}+k_{\|}\right)}{\sqrt{\tilde{\omega}^{2}-\tilde{v}_{2}^{2}\left(k_{\|}^{\prime}+k_{\|}\right)^{2}}}(13)
$$

leading to

$$
\tilde{v}_{2}-v_{2} \simeq-\Gamma_{0} \frac{\tilde{v}_{2}}{\sqrt{\Gamma_{e l}^{2}+\tilde{v}_{2}^{2} \kappa^{2}}}
$$

This result, which should be solved self-consistently together with Eq. (12), obtains only in the limit where the scattering is sufficiently forward such that the momentum integration takes place only near the node; it interpolates correctly to the isotropic limit $\kappa \rightarrow \infty$, however, where

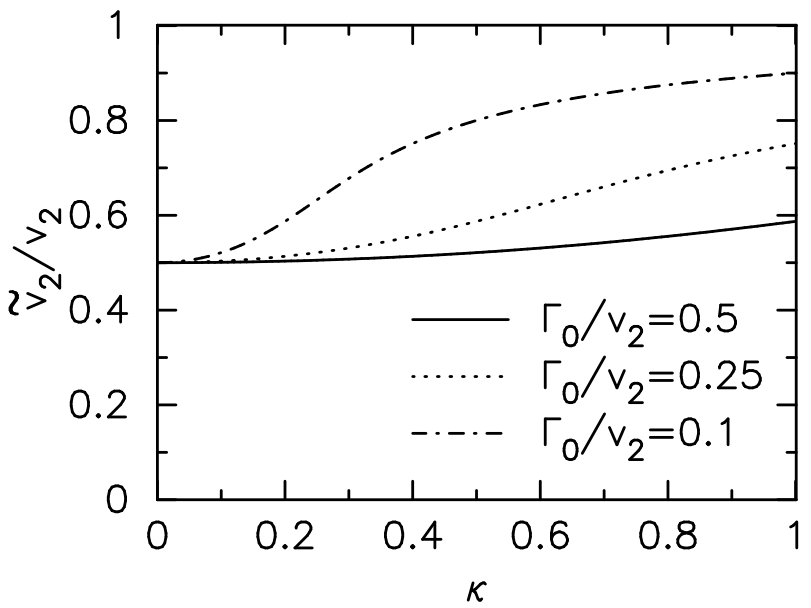

FIG. 2: Gap slope renormalization $\tilde{v}_{2} / v_{2}$ vs. inverse scattering range $\kappa$ from the solution of Eqs. (12) and (14).

the gap velocity renormalization vanishes, $\tilde{v}_{2}=v_{2}$. In the forward scattering limit $\kappa \rightarrow 0$, it is easy to check that $\tilde{v}_{2} \rightarrow v_{2} / 2$, and the dependence for small $\kappa$ is otherwise weak, as shown in Fig. 2

We will consider two different ways of characterizing the scattering rates of quasiparticles with $k_{x}=k_{y}$. First, suppose $\mathbf{k}$ is fixed exactly at the nodal Fermi surface $\mathbf{k}_{N}$. In this case the quasiparticle energy $\omega=0$ and one can study $\Gamma_{e l}\left(\mathbf{k}_{N}, \omega=0\right)$ as a function of temperature. The contribution of the out-of-plane elastic impurity scattering to the temperature dependence of the MDC line width $\Delta k(T)$ for a quasiparticle with $\mathbf{k}=\mathbf{k}_{N}$ is given by $2 \Gamma_{e l}\left(\mathbf{k}_{N}, \omega=0\right) / v_{F}$. When $\omega=0$, Eq. (12) can be written as

$$
1=\frac{\Gamma_{0}}{\tilde{v}_{2} \kappa} \ln \left(\frac{1+\sqrt{1+\left(\Gamma_{e l} / \tilde{v}_{2} \kappa\right)^{2}}}{\left(\Gamma_{e l} / \tilde{v}_{2} \kappa\right)}\right) .
$$

At low temperatures, if $\tilde{v}_{2} \kappa / \Gamma_{0} \gg 1$, we find the familiar Born result

$$
\Gamma_{\mathrm{el}}(T) \simeq \tilde{v}_{2} \kappa e^{-\left(\frac{\tilde{v}_{2} \kappa}{\Gamma_{0}}\right)}
$$

with the gap maximum $\Delta_{0}$ in the isotropic case replaced by $\tilde{v}_{2} \kappa$. When $\tilde{v}_{2} \kappa / \Gamma_{0} \ll 1$,

$$
\Gamma_{\mathrm{el}}(T) \simeq \Gamma_{0}\left(1-\frac{1}{6}\left(\frac{\tilde{v}_{2} \kappa}{\Gamma_{0}}\right)^{2}\right)
$$

This limit, of course, always applies when $T$ goes to $T_{c}$, but it can also apply for all values of $T / T_{c}$ if the system is sufficiently dirty. Note that since $\Gamma_{0}$ varies as $\left|V_{0}\right|^{2} \kappa$, if $V_{0}$ is kept fixed, the normal state scattering rate decreases as $\kappa$ becomes smaller. This simply reflects the reduction of phase space as the scattering is restricted to be in a more narrow forward cone. At the same time, the drop in the scattering rate as the gap $\Delta_{0}(T)$ opens for $T<T_{c}$, is smaller for reduced values of $\kappa$. This reflects the fact that the maximum effective gap reached by a scattered 

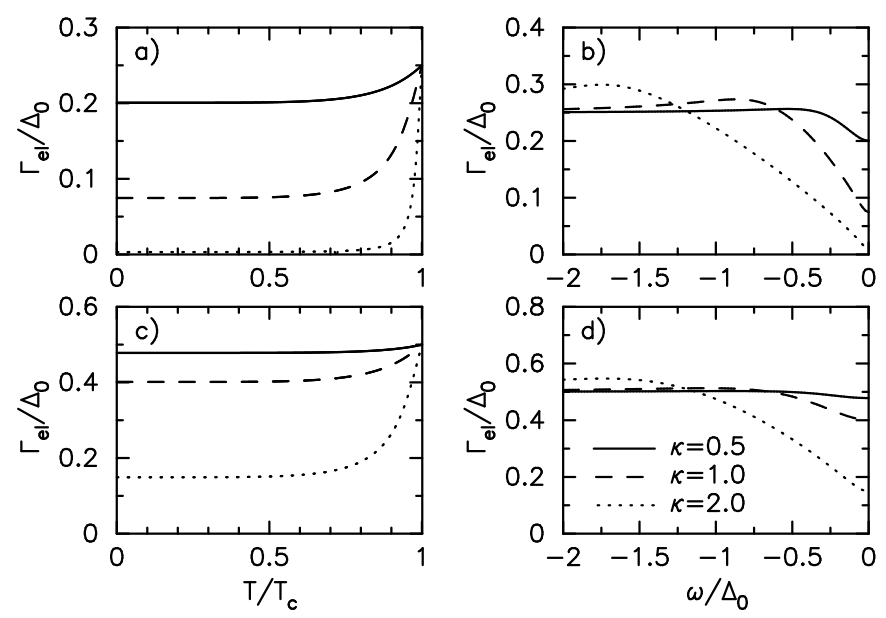

FIG. 3: Evaluation of the elastic nodal scattering rate $\Gamma_{\mathrm{el}}$ from Eqs. (12) and (14). a) temperature dependence of $\Gamma_{\mathrm{el}}(\omega=0, T)$ for three values of the impurity potential range parameter $\kappa=0.5$ (solid), 1.0 (dashed), and 2.0 (dotted) with $\left.\Gamma_{0}=0.25 \Delta_{0} ; \mathrm{b}\right)$ energy dependence of $\Gamma_{\mathrm{el}}(\omega, T=0)$ for the same parameters; c),d) same as a),b) but for $\Gamma_{0}=0.5 \Delta_{0}$.

nodal quasiparticle is reduced by $\kappa$ so that the opening of the gap is less effective in suppressing the scattering rate. Results obtained from solving Eqs. (12) and (14) for $\Gamma_{0} / \Delta_{0}=0.5$ and 0.25 are shown in Fig. (3 a) and c) as a function of $\kappa$. The temperature dependence of the elastic scattering arises from the temperature dependence of the $d$-wave gap, and therefore varies as $T^{3}$ at low $T$. Note that the interpolation form, Eq. (9), actually gives a qualitatively incorrect result at low temperatures for a $d$-wave superconductor. Eq. (9) implies an activated thermal depletion of the condensate, whereas the correct solution to the $d$-wave gap equation has a leading term varying as $T^{3}$ 16], giving $\Delta_{0}(T) \simeq \Delta_{0}\left(1-b T^{3} / \Delta_{0}^{3}\right)$, where $b$ is a constant of order unity. In the clean limit, it follows from Eq. (16) that

$$
\begin{aligned}
\Gamma_{\mathrm{el}}(T) & \simeq \Gamma_{\mathrm{el}}(T=0)\left(1+\frac{b\left(\tilde{v}_{2} \kappa-\Gamma_{0}\right)}{\Delta_{0}^{3} \Gamma_{0}} T^{3}\right) \\
& \simeq \Gamma_{\mathrm{el}}(T=0)\left(1+2 \frac{b \kappa T^{3}}{\Gamma_{0} \Delta_{0}^{2}}\right)
\end{aligned}
$$

while in the dirty limit, Eq. (17) implies that

$$
\begin{aligned}
\Gamma_{\mathrm{el}}(T) & \simeq \Gamma_{0}\left(1-\frac{\left(\tilde{v}_{2} \kappa\right)^{2}}{6 \Gamma_{0}^{2}}\right)+\frac{b\left(\tilde{v}_{2} \kappa\right)^{2}}{3 \Delta_{0}^{3} \Gamma_{0}} T^{3} \\
& \simeq \Gamma_{0}\left(1-\frac{\Delta_{0}^{2} \kappa^{2}}{6 \Gamma_{0}^{2}}\right)+\frac{b \kappa^{2}}{3 \Delta_{0} \Gamma_{0}^{2}} T^{3} .
\end{aligned}
$$

In Eqs. (18) and (19) we have assumed $v_{2} \simeq 2 \Delta_{0}$ and used the asymptotic results for $\tilde{v}_{2} / v_{2}$ derived above to obtain simple expressions for the clean and dirty limits, respectively.

It is also interesting to study the $\omega$ energy dependence of $\Gamma_{e l}$ at a fixed temperature. Within the current ap- proximate framework represented by Eqs. (12) and (14), we will study $\Gamma_{\mathrm{el}}\left(\omega, \mathbf{k}_{N}, T\right)$. For $T$ small compared with $T_{c}$, one obtains the results shown in Fig. $3 \mathrm{~b}$ ) and d). The initial increase in the scattering rate as the binding energy $|\omega|$ increases reflects the opening of the nodal phase space with increased quasiparticle energy. When $|\omega|$ is large compared to $\tilde{v}_{2} \kappa$, the scattering saturates at its normal state value. By expanding Eq. (12), we find that

$$
\Gamma_{\mathrm{el}}(\omega) \simeq \Gamma_{0}\left(1+\frac{1}{6}\left(\frac{\tilde{v}_{2} \kappa}{\omega}\right)^{2}\right)
$$

so this saturation actually occurs from values of $\Gamma_{e l}$ somewhat above $\Gamma_{0}$, as seen in Fig. 3

For forward scattering, where $\tilde{v}_{2} \kappa / \Gamma_{0}$ is small, one can obtain a useful estimate of the quasilinear slope. In this case, $\Gamma_{\mathrm{el}}(\omega)$ rises from its value of $\Gamma_{\mathrm{el}}(\omega=0)$ at $\omega=0$ to $\Gamma_{0}$ over a frequency range of order $\tilde{v}_{2} \kappa$ with a quasilinear slope $\left(\Gamma_{0}-\Gamma_{\mathrm{el}}(\omega=0)\right) /\left(\tilde{v}_{2} \kappa\right)$. In the dirty limit, Eq. (17), for $0<|\omega|<\tilde{v}_{2} \kappa$ this gives

$$
\Gamma_{\mathrm{el}}(\omega) \simeq \Gamma_{\mathrm{el}}(\omega=0)+\frac{1}{6}\left(\frac{\tilde{v}_{2} \kappa}{\Gamma_{0}}\right) \omega .
$$

Finally, if the weak scattering is sufficiently isotropic $(\kappa \gtrsim 1)$, we recover the standard result for pointlike weak scatterers

$$
\Gamma_{\mathrm{el}}(\omega) \simeq \Gamma_{\mathrm{el}}(\omega=0)+\frac{\pi}{2} \frac{n_{i} N_{0}\left|V_{0}\right|^{2} \omega}{\Delta_{0}} .
$$

Which of the results, Eq. (21) or 221) is valid depends upon the character of the disorder.

To check these estimates, and evaluate the scattering rate for various scattering potential ranges and impurity concentrations, as well as to treat momenta away from the Fermi surface more accurately, we introduce a more realistic model for the elastic scattering. We consider a screened exponential fall-off in $2 \mathrm{D}$ such that,

$$
\left|V\left(\mathbf{k}, \mathbf{k}^{\prime}\right)\right|^{2}=\frac{\left|V_{0}\right|^{2}}{\mathbf{q}^{2}+\kappa^{2}}
$$

where $\mathbf{q}=\mathbf{k}-\mathbf{k}^{\prime}$ is the momentum transfer. We then perform a self-consistent solution of equations (5) and (6). Results for $\Gamma_{\mathrm{el}}$ analogous to Fig. [3 but taken "on-shell" at momentum $\mathbf{k}$ such that $\varepsilon_{\mathbf{k}}=\omega$ are presented in Fig. [4 Results have been scaled such that the (self-consistent) normal state scattering rate is the same for all curves; as discussed above, this implies that the more forward scattering cases correspond to larger impurity concentrations. It is seen that the qualitative behavior close to the Fermi level is captured rather well by the approximate model discussed above where the scattering is restricted to a cone of width $\sim \kappa$. It is also interesting to note that the quasilinear behavior at the low $\omega$ values persists until extremely long scattering ranges, of order 5-10 lattice spacings, for reasonable total normal state scattering rates. This suggests on the one hand that elastic 

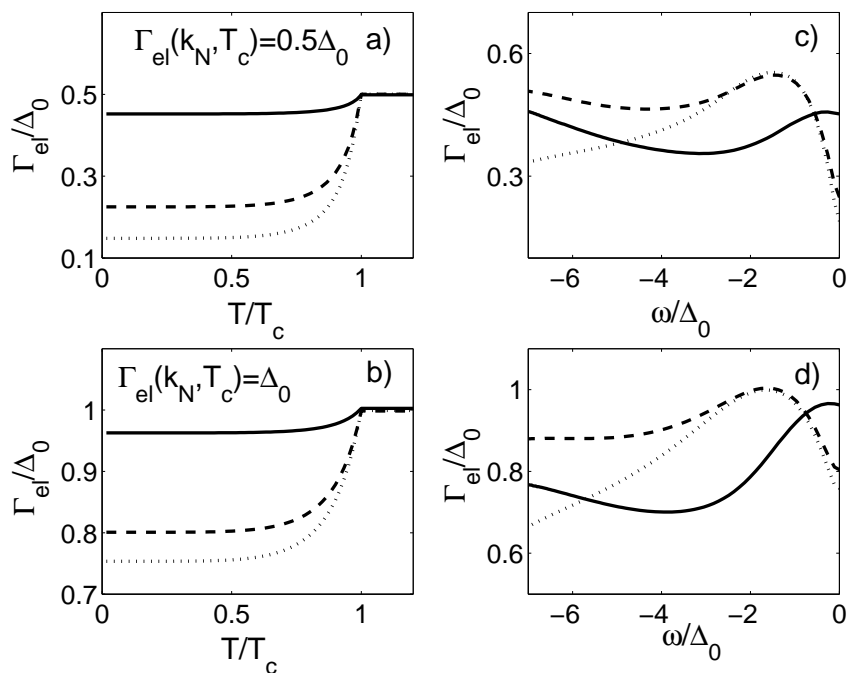

FIG. 4: Elastic scattering rate $\Gamma_{\mathrm{el}}$ for the Yukawa-type potential with range $\kappa^{-1}$ Eq. (23). a): $\Gamma_{\text {el }} / \Delta_{0}$ vs. $T / T_{c}$ for a normal state elastic scattering rate $\Gamma_{\mathrm{el}}\left(k_{N}, T_{c}\right)=0.5 \Delta_{0}$ and $\kappa=0.1,1$, and $\infty$ (isotropic). c): $\Gamma_{\mathrm{el}} / \Delta_{0}$ vs. $\omega / \Delta_{0}$ for the same $\kappa$ and $\Gamma_{\text {el }}\left(k_{N}, T_{c}\right)$ values. b) and d): same quantities plotted as above, but with $\Gamma_{\mathrm{el}}\left(k_{N}, T_{c}\right)=\Delta_{0}$. Results for different values of the scattering parameter $\kappa=0.1$ (solid), 1 (dashed), and the isotropic case $\kappa=\infty$ (dotted) are shown.

scattering is a likely explanation for the quasilinear behavior seen at low energies in the MDC width; on the other hand, it also means that it will be difficult to determine the range of the scatterers precisely from such a measurement alone.

\section{INELASTIC SCATTERING}

In this section we discuss the scattering rates due to inelastic electron-electron scattering by a phenomenological short range Coulomb interaction based on the two dimensional Hubbard model. For the tight-binding bandstructure considered here, these scattering processes are dominated by exchange of antiferromagnetic spin-fluctuations. We are using a conventional BerkSchrieffer-like [19] theory as has been used before to discuss the single-particle inelastic lifetime [20] and has proved to give a qualitative description of low-energy microwave and thermal conductivity lifetimes [21, 22] as well as NMR relaxation rates 23] in the cuprates.

In second-order perturbation theory for the two dimensional Hubbard model, the imaginary part of the nodal quasiparticle self-energy due to inelastic scattering from the onsite Coulomb interaction $U$ can be written as

$$
\begin{aligned}
& \Gamma_{\text {inel }}(\omega, \mathbf{k}, T)=-\Sigma_{\text {inel }}^{\prime \prime}(\omega, \mathbf{k}, T)= \\
& \quad \frac{U^{2}}{N} \sum_{q} \int_{-\infty}^{\infty} d \Omega[n(\Omega)+f(\Omega-\omega)] . \\
& \quad \chi_{0}^{\prime \prime}(q, \Omega) N(k-q, \omega-\Omega)
\end{aligned}
$$

with

$$
N(k, \omega)=-\frac{1}{\pi} \operatorname{Im}\left(\frac{\omega+i \delta+\varepsilon_{k}}{(\omega+i \delta)^{2}-E_{k}^{2}}\right)
$$

and

$$
\begin{aligned}
& \chi_{0}(\mathbf{q}, \Omega)= \\
& \quad \frac{1}{N} \sum_{k}\left\{\frac{1}{2}\left[1+\frac{\varepsilon_{k+q} \varepsilon_{k}+\Delta_{k+q} \Delta_{k}}{E_{k+q} E_{k}}\right] \frac{f\left(E_{k+q}\right)-f\left(E_{k}\right)}{\Omega-\left(E_{k+q}-E_{k}\right)+i 0^{+}}\right. \\
& +\frac{1}{4}\left[1-\frac{\varepsilon_{k+q} \varepsilon_{k}+\Delta_{k+q} \Delta_{k}}{E_{k+q} E_{k}}\right] \frac{1-f\left(E_{k+q}\right)-f\left(E_{k}\right)}{\Omega+\left(E_{k+q}+E_{k}\right)+i 0^{+}} \\
& \left.+\frac{1}{4}\left[1-\frac{\varepsilon_{k+q} \varepsilon_{k}+\Delta_{k+q} \Delta_{k}}{E_{k+q} E_{k}}\right] \frac{f\left(E_{k+q}\right)+f\left(E_{k}\right)-1}{\Omega-\left(E_{k+q}+E_{k}\right)+i 0^{+}}\right\}(26)
\end{aligned}
$$

Here, $E_{k}=\sqrt{\varepsilon_{k}^{2}+\Delta_{k}^{2}}$. As before, we are considering a nodal quasiparticle with $k_{x}=k_{y}$ and the magnitude of $k$ is set by $\omega=\varepsilon_{k}$. At low energies, where $\omega$ and $T$ are small compared to the zero temperature gap amplitude, Eq. (24) determines the form of the $\omega$ and $T$ dependence of the inelastic quasiparticle scattering. Higher order processes lead to a renormalization of $U$, but the phase space restrictions imposed by the $d$-wave nature of the superconducting state determine the low $T$ and $\omega$ dependence of $\Gamma_{\text {inel }}\left(\omega,\left.\mathbf{k}\right|_{\omega=\varepsilon_{\mathbf{k}}}, T\right)$ [20, 21, 24].

For $\mathbf{k}=\mathbf{k}_{N}$ and $\omega=0$, the low temperature inelastic scattering varies as

$$
\Gamma_{\text {inel }}(0, T) \simeq \frac{T^{3}}{\Delta_{0}^{2}}
$$

with a prefactor of order one 20, 24]. For the set of parameters discussed below the prefactor becomes 2.4. It has been argued that $\Gamma_{\text {inel }}$ should vary as $T^{5 / 2}$ at low temperatures 25]. However, for the temperatures $T>$ $0.05 T_{c}$ we have studied, the $T^{3}$ behavior provides a much better fit to our numerical results in Fig. [6). Similarly, at $T=0$ the $\omega$ dependence is given by

$$
\Gamma_{\text {inel }}(\omega, 0) \simeq \frac{\omega^{3}}{\Delta_{0}^{2}} .
$$

The extra power $T^{3}$ and $\omega^{3}$ versus the $T^{2}$ and $\omega^{2}$ dependence of the usual (3D) Fermi liquid inelastic quasiparticle Coulomb scattering is due to the $\omega / \Delta_{0}$ dependence of the $d$-wave density of states. Note that the usual $T^{3}$ and $\omega^{3}$ phonon contributions to the low energy quasiparticle scattering in a normal metal give contributions which vary as $T^{4}$ and $\omega^{4}$ in a $d$-wave superconductor.

At higher energies, the problem becomes more complicated. New, collective channels may open such as the so-called $\pi$-resonance or possibly the $B_{1 g}$ phonon. Here, we replace $\chi_{0}(q, \omega)$ by the RPA form

$$
\chi(q, \omega)=\frac{\chi_{0}(q, \omega)}{1-U \chi_{0}(q, \omega)}
$$

and consider the spin-fluctuation contributions to the scattering of the nodal quasiparticle. Following the usual 


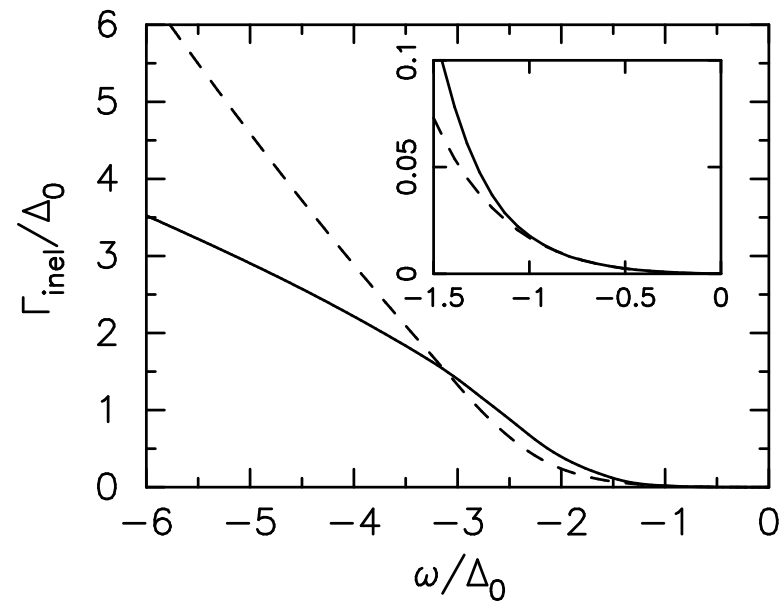

FIG. 5: Comparison of on-shell spin-fluctuation inelastic scattering rates for $\Gamma_{\text {inel }}\left(\omega,\left.\mathbf{k}\right|_{\omega=\varepsilon_{\mathbf{k}}}, T\right) / \Delta_{0}$ vs. $\omega / \Delta_{0}$ for a reduced temperature $T / T_{c}=0.1$. Dashed line: 2nd order perturbation theory with $U=6.7 t$. Solid line: RPA theory with $U=2.2 t$. Inset shows magnified low energy region.

spin-fluctuation notation, we replace the coupling $U^{2}$ in Eq. (24) by $\frac{3}{2} U^{2}$ which in any case is simply a phenomenological coupling constant in these calculations. In principle, the $U$ that enters the coupling is different from the effective $U$ in the denominator of $\chi$, Eq. 29] because of vertex corrections. Here, we will for simplicity ignore this distinction so we have just the basic RPA form of the spin-fluctuation interaction. A similar approach was used to discuss the microwave and thermal conductivity lifetimes 21]. This approach will in principle include resonant spin excitations like those which have been proposed to explain the $\pi$ resonance, i.e. the peak seen by neutron scattering at around $40 \mathrm{meV}$ in various cuprates, but we do not investigate these effects in detail here. Rather, we will examine what this approximation gives for the higher energy dependence of $\Gamma_{\text {inel }}\left(\omega,\left.\mathbf{k}\right|_{\omega=\varepsilon_{\mathbf{k}}}, T\right)$ numerically.

In Fig. 5. we show the numerical evaluation of Eq. (24), as well as the RPA result with $\chi_{0}$ replaced by $\chi$ as in Eq. (29), using the band parameters discussed above in Eqs. (77) and [8) appropriate for an optimally doped cuprate. Here, the numerical integrations in Eq. (24) and (26) are done using the technique described in Ref. [26]. The value of $U=2.2 t$ chosen in the RPA case comes from early fits of spin-fluctuation theory to NMR data, and more recently to microwave and thermal conductivity data on YBCO, but is expected to be reasonable for BSCCO as well because transport data suggest that the inelastic scattering rates at $T_{c}$ are very similar. The value of $U=6.7 t$ in the case of the second order perturbation theory result was chosen so that the $\omega^{3}$ behavior of both results matches for $|\omega| / \Delta_{0}<1$ as shown in the inset. As noted, when $|\omega| \lesssim \Delta_{0}$, the $\omega$-dependence of the inelastic scattering is determined by the phase space, and one gets an $\omega^{3}$ dependence from a constant $U$ interaction as well as from the spin fluctuation (RPA) interaction. However, as shown in Fig. [5] at higher energies the scattering
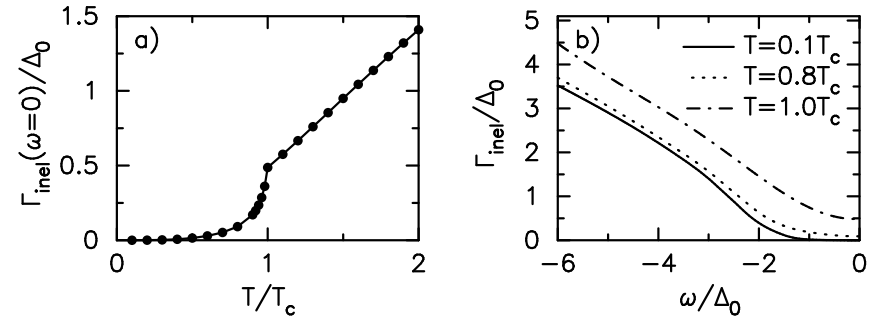

FIG. 6: Left panel: Temperature dependence of inelastic RPA spin fluctuation scattering rate $\Gamma_{\text {inel }}\left(0, \mathbf{k}_{N}, T\right) / \Delta_{0}$ vs. $T / T_{c}$ for $U=2.2 t$. Right panel: Frequency dependence $\Gamma_{\text {inel }}\left(\omega,\left.\mathbf{k}\right|_{\omega=\varepsilon_{\mathbf{k}}}, T\right) / \Delta_{0}$ vs. $\omega / \Delta_{0}$ for reduced temperatures $T / T_{c}=0.1,0.8$, and 1.0 .

reflects the frequency dependence of the effective interaction. In the RPA case, the classical quasilinear energy dependence expected in spin fluctuation theory when the energy exceeds the spin-fluctuation energy is clearly visible, and the crossover to the low-energy $\omega^{3}$ form takes place around $3 \Delta_{0}$, as also found in Ref. 22].

In Figures 6] a) and b), we study the effect of temperature on the inelastic scattering. First, in Fig. 6 a) we consider the nodal scattering rate at $\omega=0$, which collapses rapidly at $T_{c}$ due to the opening of the gap. In Fig. [ b), the energy dependence of the scattering rate is plotted for various temperatures. It is interesting to note that for these band parameters, there is a considerable amount of upward curvature at low $\omega$ in $\Gamma_{\mathrm{el}}\left(\omega,\left.\mathbf{k}\right|_{\omega=\varepsilon_{\mathbf{k}}}, T\right)$ even at $T_{c}$, in contrast to the simpler nearly nested bands considered in [20, 22].

\section{TOTAL SCATTERING RATE}

To include both types of scattering effects, we neglect interference processes between electron-electron collisions and impurity scattering entirely, and approximate the total scattering rate at the node by

$$
\Gamma_{\text {tot }}=\Gamma_{\text {el }}+\Gamma_{\text {inel }},
$$

i.e. higher order processes like the influence of the inelastic scattering on the elastic scattering and vice versa are neglected. However, this is partially taken into account since we have chosen the parameters for the elastic and inelastic scattering on a phenomenological basis.

In Fig. 7 we plot the on-shell total scattering rate $\Gamma_{\text {tot }}$ of a nodal quasiparticle as a function of both temperature and energy. It is seen that the generic features, for reasonable assumptions about the magnitudes of the impurity scattering rates and ranges, are as follows. As a function of temperature, one expects the nodal scattering rate to collapse at $T_{c}$, and to obey a $T^{3}$ dependence at the lowest temperatures, whose coefficient is determined by both the elastic and inelastic processes. On the other hand, the low energy dependence of the scattering rate 

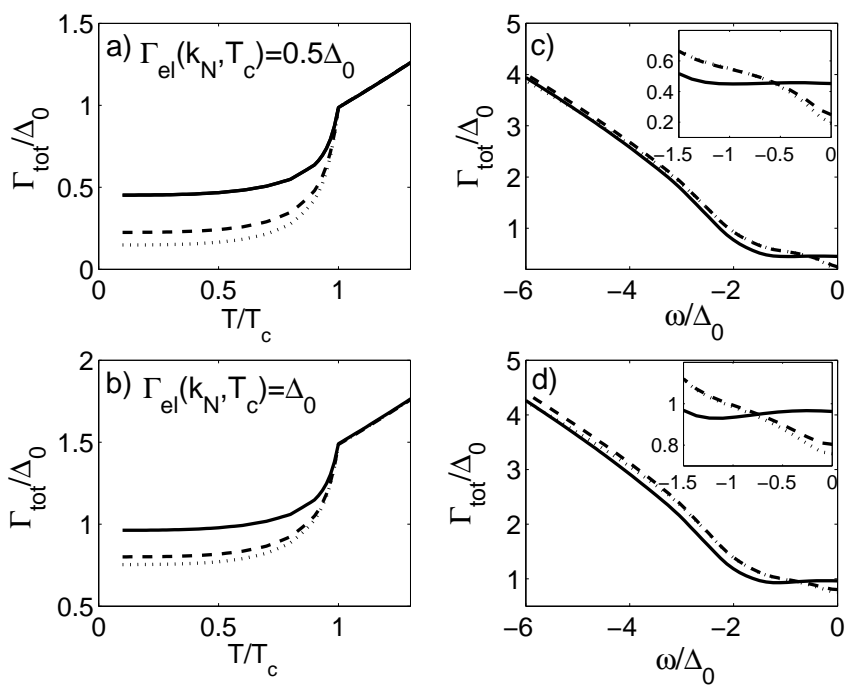

FIG. 7: a) and b): Temperature dependence of the total scattering rate $\Gamma_{\text {tot }}\left(0, k_{N}, T\right) / \Delta_{0}$ vs. $T / T_{c}$ for $U=2.2 t$ and $\Delta_{0} / t=0.2$, and normal state elastic scattering rates of $\Gamma_{\mathrm{el}}\left(0, k_{N}, T_{c}\right) / \Delta_{0}=0.5$ and 1 , respectively. c) and d): Frequency dependence of the on-shell scattering rate $\Gamma_{\text {tot }}\left(\omega,\left.k\right|_{\omega=\varepsilon_{k}}\right) / \Delta_{0}$ vs. $\omega / \Delta_{0}$ for the same $U$ and $\Delta_{0}$ and $T=0.1 T_{c}$ with $\Gamma_{\mathrm{el}}\left(0, k_{N}, T_{c}\right) / \Delta_{0}=0.5$ and 1 , respectively. Results for different values of $\kappa=0.1$ (solid), 1 (dashed), and $\kappa=\infty$ (dotted) are shown.

at low temperatures is dominated by the quasi-linear $\omega$ dependence of the elastic scattering. The generic result appears to be a quasilinear low-energy behavior for weak out of plane impurities, where the slope of this result is unrelated to the slope of the high-energy quasilinear behavior determined by spin-fluctuation scattering. A very low-energy $(\omega \sim 1-2 \mathrm{meV}$ ) flattening or upturn due to unitary scatters $\left(\sum_{\mathbf{k}} G \simeq \omega\right)$ at low energies in Eq. (3), is expected to be present in principle. This contribution is likely to be negligible in BSCCO due to the small concentration of native planar defects in current samples, and the fact that the expected impurity bandwidth is comparable to the energy resolution in ARPES experiments.

\section{CONCLUSIONS}

Understanding the lifetime of nodal quasiparticles is a fundamental problem of cuprate physics which must be solved if we are to agree that a $d$-wave BCS theory describes the optimally to over-doped superconducting state, the only part of the phase diagram apparently susceptible to semiquantitative description at this time. In addition, the fact that nodal quasiparticles appear to be robust even for strongly underdoped samples may be an important clue to the physics of the pseudogap, and a theory of the nodal states within BCS which works at optimal doping may help us to understand this clue. Finally, the lifetime of the nodal quasiparticles determines thermal and microwave conductivity as well as photoemission lineshapes and one would like to have a unified description of these quantities within a single model. Here, we have calculated the on-shell $\omega=\varepsilon_{k}$ nodal quasiparticle scattering rate which enters in determining the MDC linewidth. Using a simple model which parameterizes the forward elastic scattering in terms of a range $\kappa^{-1}$, a normal state scattering rate $\Gamma_{0}$, and an inelastic spin-fluctuation scattering parameterized by an RPA form with an effective Coulomb coupling $U$, we have studied the temperature and $\omega$-dependence of the scattering rate.

For a quasiparticle at the nodal momentum $k_{N}$, as $T$ decreases below $T_{c}$, the elastic scattering rate decreases from its normal state value $\Gamma_{0}$. As the gap opens, it reaches a smaller value $\Gamma_{\mathrm{el}}(T)$ determined by the forward scattering parameter $\kappa$ and the renormalized gap velocity $\tilde{v}_{2}$. This temperature dependence of the elastic scattering rate arises from the temperature dependence of the gap amplitude which controls the available phase space for scattering, suppressing it as the gap opens. Because the scattering rate depends exponentially on the gap, which opens rapidly in BSCCO, the elastic scattering rate has a cusp-like onset at $T_{c}$, decreasing rapidly to its low temperature value. The inelastic contribution to scattering rate also decreases as the gap opens, with a cusp, and then decreases as $\left(T / \Delta_{0}\right)^{3}$ at low temperatures.

At low reduced temperatures, the $\omega$-dependence of the elastic scattering rate can exhibit a quasi-linear behavior, varying as $\frac{\omega}{6}\left(\frac{\tilde{v}_{2} \kappa}{\Gamma_{0}}\right)$ if the scattering is forward or as $\Gamma_{0} \frac{\omega}{\Delta_{0}}$ if the scattering is more isotropic. The inelastic scattering rate initially increases as $\omega^{3} / \Delta_{0}^{2}$ so that there is an energy beyond which the inelastic scattering becomes dominant. For energies greater than of order $3 \Delta_{0}$, the inelastic scattering rate crosses over to a quasi-linear $\omega$-dependence with a slope of order one.

Although the calculations we have presented here are straightforward, at the present time the experimental situation regarding the direct measurement of the nodal scattering rate by ARPES is somewhat uncertain. There are some claims in the literature that the rate collapses in the SC state [9], as found theoretically here, and some that marginal Fermi liquid linear behavior consistent with a quantum critical point persists down to the lowest temperatures [ 8$]$. As samples and resolution of the ARPES technique improve, we expect this discrepancy to be resolved and our prediction for the nodal quasiparticle MDC width to be testable.

\section{Acknowledgments}

The authors are grateful for discussions with D. Dessau, A. Fujimori, A. Ino, P. Johnson, and Z.X. Shen. We would like to thank A. Chubukov for his comments regarding the second order on-shell inelastic scattering rate. Work was partially supported by ONR 
grant N00014-04-0060 and NSF DMR02-11166.

[1] Y. Zhang, N.P. Ong, P.W. Anderson, D.A. Bonn, R. Liang, and W.N. Hardy, Phys. Rev. Lett. 86, 890 (2001).

[2] M. Chiao, R.W. Hill, C. Lupien, B. Popic, R. Gagnon, and L. Taillefer, Phys. Rev. Lett. 82, 2943 (1999).

[3] A. Hosseini, R. Harris, S. Kamal, P. Dosanjh, J. Preston, R. Liang, W.N. Hardy, and D.A. Bonn, Phys. Rev. B 60, 1349 (1999).

[4] P.J. Hirschfeld, W.O. Putikka, and D.J. Scalapino, Phys. Rev. B 50, 10250 (1994).

[5] M.B. Walker and M.F. Smith, Phys. Rev. B 61, 11285 (2000).

[6] A.C. Durst and P.A. Lee, Phys. Rev. B 62, 1270 (2000).

[7] D. Dessau, J.D. Koralek, private communication; A. Fujimori, private communication; A. Ino, M. Taniguchi, private communication; P. Johnson, T. Valla, private communication.

[8] T. Valla, A.V. Fedorov, P.D. Johnson, Q. Li, G.D. Gu, and N. Koshizuka, Phys. Rev. Lett. 85, 828 (2000).

[9] A. Kaminski, J. Mesot, H. Fretwell, J.C. Campuzano, M.R. Norman, M. Randeria, H. Ding, T. Sato, T. Takahashi, T. Mochiku, K. Kadowaki, and H. Hoechst Phys. Rev. Lett. 84, 1788 (2000).

[10] A. A. Kordyuk, S. V. Borisenko, A. Koitzsch, J. Fink, M. Knupfer, B. Büchner, H. Berger, G. Margaritondo, C. T. Lin, B. Keimer, S. Ono, and Yoichi Ando, Phys. Rev. Lett. 92, 257006 (2004).

[11] E. Abrahams and C.M. Varma, Proc. Nat'l Acad. Sci. 97, 5714 (2000).

[12] L. Zhu, P.J. Hirschfeld, and D.J. Scalapino, Phys. Rev. B 70, 214503 (2004).

[13] H. Eisaki, N. Kaneko, D.L. Feng, A. Damascelli,
P.K. Mang, K.M. Shen, Z.-X. Shen, and M. Greven, Phys. Rev. B 69, 064512 (2004).

[14] E.W. Hudson, V. Madhavan, K. McElroy, J.E. Hoffman, K.M. Lang, H. Eisaki, S. Uchida, and J.C. Davis, Physica B 329, 1365 (2003).

[15] For a recent treatment of higher-order processes in the forward scattering limit, see C.T. Rieck, K. Scharnberg, S. Scheffler; cond-mat/0408320

[16] See, e.g. H. Won, S. Haas, D. Parker, S. Telang, A. Vanyolos, and K. Maki; cond-mat/0501463

[17] H.-Y. Kee, Phys. Rev. B 64, 012506 (2001).

[18] See also, G. Haran and A.D.S. Nagi, Phys. Rev. B 54, 15463 (1996); ibid 58, 12441 (1998); M.L. Kulic and O.V. Dolgov, Phys. Rev. B 60, 13062 (1999).

[19] N. F. Berk and J. R. Schrieffer, Phys. Rev. Lett. 17, 433 (1966).

[20] S.M. Quinlan, D.J. Scalapino, and N. Bulut, Phys. Rev. B 49, 1470 (1994).

[21] D. Duffy, P.J. Hirschfeld, and D.J. Scalapino, Phys. Rev. B 64, 224522 (2001).

[22] S.M. Quinlan, P.J. Hirschfeld, and D.J. Scalapino, Phys. Rev. B 53, 8575 (1996).

[23] N. Bulut and D.J. Scalapino, Phys. Rev. B 45, 2371 (1992).

[24] M.L. Titov, A.G. Yashenkin, and D.N. Aristov, Phys. Rev. B 52, 10626 (1995).

[25] J. Paaske and D.V. Khveshchenko, Physica C 341-348, 265 (2000).

[26] T. Dahm and L. Tewordt, Phys. Rev. Lett. 74, 793 (1995); Phys. Rev. B 52, 1297 (1995). 